\section{Concept to Sale of a Healthier Snack Bar}

\author{
Elaine C. Rush*, Mary R. Yan, Andrew Parsons, John Kelleher, Deborah MacRae, David Brown
}

AUT Food Network, Auckland University of Technology, Auckland, New Zealand

*Corresponding author: Elaine C. Rush, Auckland University of Technology, Auckland 0627, PO Box 92006, New Zealand, E-mail: erush@aut.ac.nz

\begin{abstract}
Snacking between meals provides an important contribution to dietary intake of key nutrients. There is a need for snack bars that are nutrient dense, have a low glycemic load and increase feelings of fullness and satiety. Over the last three years a branded "Nothing Else" dates and almonds life saver snack bar, without preservatives or additives, has been developed in partnership with a food manufacturer. In June 2015 the final bar, with eight simple ingredients, was first produced by our industry partner to meet the nutritional criteria for a health claim. Shelf-life, glycemic index, effect on appetite and satiety and market demand within retail outlets at the Auckland University of Technology were measured. More than 3000 bars were sold in five months and shelf-life exceeds four months. Anecdotal feedback is positive and new retail outlets are under consideration. Proof-of-concept and proof-of-principle for the feasibility of development of a food product by a University in partnership with the food industry has been shown. The translation of the diverse research skills and understanding found within a University to the production and successful retail of a food product is one small step towards providing a food environment that is supportive of a healthier diet.
\end{abstract}

Received Date: January 13, 2016

Accepted Date: January 27, 2016

Published Date: February 02, 2016

Citation: Rush, E.C.,et al. Concept to Sale of a Healthier Snack Bar. (2016) Int J Food Nutr Sci 3(1): 195-198.

Keywords: Branding; Food environment; Glycemic index; Health claims; Satiety; Snacks

\section{Introduction}

As identified in the Foresight report on obesity ${ }^{[1]}$ there is a need for inter-sectorial contributions to the development of healthier food environments and products. For packaged foods two of the myriad actions to do this are one: food labels that allow consumers to make an informed choice and two: reformulation of food products so that they are lower calorie and more nutrient dense.

Packaging of foods as a marketing tool is important ${ }^{[2]}$. Elements of visual branding ${ }^{[3]}$ and front-of-pack communication about the healthiness of the product ${ }^{[4,5]}$ may combine to improve customers' dietary choices. However, very little is known about how consumers process the information and make choices, with mixed results in marketing studies ${ }^{[6,7]}$. Recent suggestions are that when consumers evaluate a single food item in a non-comparative context, objective nutrition cues that offer specific quantitative information lead to higher evaluations and intentions to purchase. Furthermore, evaluative cues, such as front-of-pack labels have a more positive impact on evaluations and purchase intentions of healthier products when evaluating multiple items simultaneously in a comparative context $t^{[8]}$. Taste and cost are the two main elements that drive choice ${ }^{[9]}$. However, overall liking and brand perception of a healthier food product was improved when consumers were informed about the branding and health related nutrition information compared with the blind state ${ }^{[10]}$.

In addition reformulation and formulation of foods to improve the nutrient density profile determines if health related claims may be made ${ }^{[11]}$, but may also influence purchase ${ }^{[12]}$. The bottom line is that sales of a product determine commercial success.

Snacking between meals provides an important contribution to dietary intake of key nutrients but may also contribute to excess energy consumption ${ }^{[13]}$. Snack bars are a relatively popular snack but often are high in sugar, fat and energy. Snack bars seek to meet the needs for indulgence but also health and wellness ${ }^{[14]}$. The majority of sales in New Zealand are through store-based retailing $(99 \%)$, mainly supermarkets $(90 \%)$ with a small proportion through vending machines $(0.2 \%)^{[14]}$. Sales in 2014 were NZD132 million. Growth in sales has been strongest for energy and nutrition bars.

Therefore, the requirement for a healthier snack bar was combined with "clean" packaging and branding to better inform 
the consumer about all the ingredients in a product on the frontof-pack label.

The initial development of the "Nothing Else" brand has been reported ${ }^{[15]}$. The Nothing Else food label lists the eight or less easily recognized ingredients on the front of pack within a circular band that has the words NOTHING ELSETM.

The research reported in this article aimed to provide evidence for the proof-of-principle that the Nothing Else bar could be produced by a commercial enterprise; that within a retail University environment repeat purchasing patterns would be sustained and that the bar would have a low glycaemic index and therefore meet the criteria to make a health claim.

\section{Methods}

This report describes an action research process, where change is introduced and the effects observed and reviewed; then adjustments are made to achieve the overall outcome. The setting was the Auckland University of Technology (AUT) and the commercial partner was AB Foods; a leading local food manufacturer. Two processes were run in parallel - the development of a healthier snack bar and testing for glycemic index (GI), satiety and shelf life. Concurrently, there were regular communications and updates between AB Foods and the University and sales patterns were monitored.

Determination of glycemic index, using a cross-over treatment design, followed the protocol of the international standard ISO26642:2010 Food products- determination of the glycemic index and recommendation for food classification. Ethics approval was provided by the AUT ethics committee. Fourteen apparently healthy participants were recruited. Exclusion criteria were diabetes, haemophilia or allergy to any of the ingredients in the bar. Signed, informed consent was gained after a full explanation and an opportunity for any questions to be asked.

Participants arrived at the laboratory after an 8-hour overnight fast. Consent was reconfirmed, questions answered and it was ensured that the participants were relaxed and warm. Fasting blood glucose concentration was measured twice, 5 minutes apart using a Hemocue Glucose Analyzer (HemoCue ${ }^{\circledR} 201$ RT, Sweden). Blood was sampled from a fingerprick with an automatic lancet and drawn into a microcuvettee (HemoCue ${ }^{\circledR}$ 201 RT Microcuvette). The two measurements were averaged to provide the baseline fasting blood glucose concentration (at T0). Immediately after the second fasting blood glucose, either a drink containing 25 grams of glucose or a weighed portion of the bar that contained the equivalent amount $(25 \mathrm{~g}$, of carbohydrate) was consumed. Which treatment was first was randomly assigned. Blood glucose concentrations were measured and recorded at 15, 30, 45, 60, 90 and 120 min after completion of consumption. At T0 and each half hour, visual analogue scales (VAS) questionnaires with a maximum score of $100 \mathrm{~mm}$ were marked to record relative satiety measures: satiety, hunger, fullness and the amount that could be eaten. On a second day the same 14 participants "crossed-over" to consume either the glucose or bar so that every participant had two measures of glucose response, one to the bar the other to glucose.

Bars were manufactured and packaged (BicorTM32MB777 Orientated Polypropylene Film, ExxonMobil, Luxembourg) by AB Foods, Auckland. The eight ingredients were, in descending order by weight, oats, dates, almonds, hon- ey, sunflower oil, flaxseed, cinnamon and salt. The nutrition profile score ${ }^{[11]}$ was zero and therefore a substantiated health claim could be made. Sales to the four cafes and three sport and fitness centers on the University campuses were recorded as the date the bars left storage at the University.

As part of the shelf life trial, the bars were stored in ambient conditions, temperature and humidity monitored continuously and bars were sampled regularly for water activity, $\mathrm{pH}$, moisture (Asure Quality, Auckland), taste and visual appearance.

The incremental area under the curve (iAUC) was calculated for each participant using the trapezoid model and the area below the baseline measurement excluded. The mean GI of the bar for each participant was calculated as the iAUC for the bar divided by the iAUC for glucose and the mean GI determined for the 14 participants. The VAS scores for satiety, hunger, fullness and the amount that could be eaten at each time point were determined by measuring the length $(\mathrm{mm})$ from the left end of the scale to the participants mark. The length of the VAS score $(\mathrm{mm})$ at baseline was subtracted from the scores at 30, 60, 90 and 120 minutes. Incremental VAS scores against time for the bar were compared to the scores for the glucose drink by plotting on the same graph. Cumulated sales were plotted against date so that the rate of selling could be visualised.

\section{Results and Discussion}

Overall mean fasting glucose for 56 measurements was $4.7 \pm 0.1$ (SEM), with no difference between treatments. The mean GI for the bar was $51 \pm 6$ (SEM), Fig. 1. The maximum blood glucose measured was 11.7 mmol.L-1 for the glucose and 7.9 mmol.L-1 for the bar. Also, there was a trend for the blood glucose at 120 minutes to be lower than the baseline glucose (Figure 1).
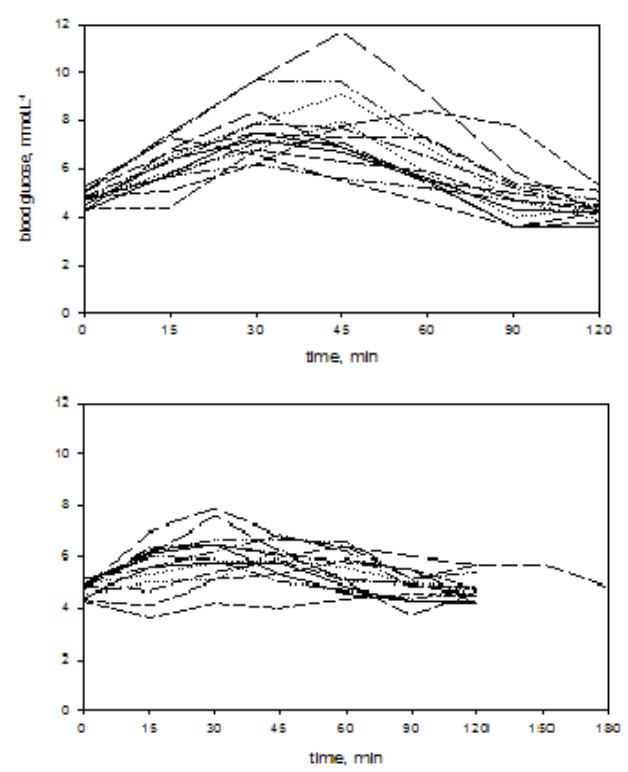

Figure 1: Glucose response curves for $25 \mathrm{~g}$ of carbohydrate and 14 participants- top: glucose bottom: bar

One participant was measured for extra time as their blood glucose concentration had not returned to baseline. Compared with the glucose drink over the 120 minutes, the Nothing Else bar was more satiating and induced a feeling of fullness while perceptions of hunger and the amount that participants 
thought that they could eat was reduced (Figure 2). At 120 minutes these differences were maintained and the satiation apparently induced by the Nothing Else bar was higher than baseline.
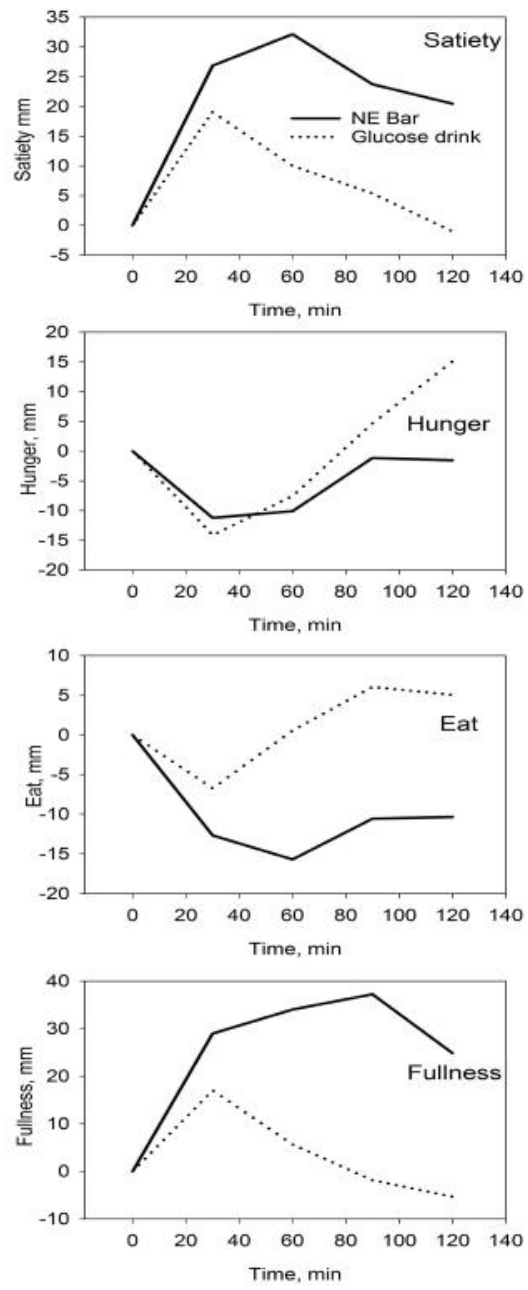

Figure 2: Satiety scores for Nothing Else bar (solid) compared with glucose drink (dots)

Sales of the bar were steady (Figure 3). Slight plateaus were seen in July and September in line with inter and intrasemester breaks of the University. Anecdotally, it was observed that many sales were for more than one bar, that it was often women making the purchase and that a number of customers returned for repeat purchases. For the outlets that also served coffee, the sales were higher. Shelf life measures are ongoing but, to date, the shelf life of the bar is four months and the water activity of the bars has remained at below 0.73 .

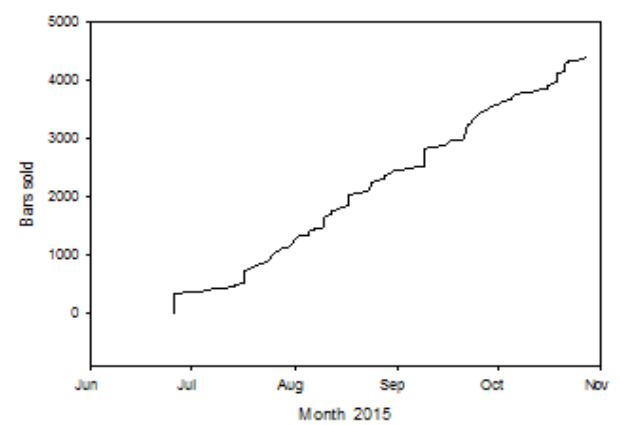

Figure 3: Cumulative sales of the Nothing Else bar in the University outlets for 4 months between July and November 2015.
This report has shown that a healthier snack bar with a low GI no additives or preservatives can be produced on commercial premises, induces greater satiety than a glucose drink and has repeat purchases over the period of 4 months.

To the best of our knowledge no other groups have reported, in the literature, this combination of outcomes or a similar process of collaboration. The "success" of the introduction of new food products to the marketplace is not easy to predict and this product has not formally been launched. The marketplace for snacks is affected by many factors including authenticity, familiarity, current trends, packaging and competition ${ }^{[16]}$ and there is a high risk of "failure". However with increased calls for reduction of intake of added sugar ${ }^{[17]}$, policies in hospitals and public institutions about healthier food environments ${ }^{[18]}$ and rising demand from consumers for healthier options in snack foods ${ }^{[19,20]}$ a case may also be made for development of products to fit a required nutrition profile and to have substantiated health claims that are part of the marketing approach ${ }^{[21]}$.

The lack of preservatives and additives meant that the bar had to be baked longer to remove free water and initially was perceived as dry by some consumers. This also meant that the energy density was increased. However, it appears that repeated consumption was associated with increased liking for the bar. Furthermore, this proof-of-principle was on a very small scale, over a short time, and within a relatively closed environment so may not be generalizable to the larger commercial market. It does, however, show that a bar, initially perceived as less palatable $^{[10]}$ can be sold successfully. With a move to clean labels ${ }^{[22]}$, whole foods and consumer and social demand for a healthier food supply it is important that product development of this type does not rely only on a perception of what the consumer wants or initial responses but that the market is tested in real time.

\section{Conclusion}

This on-going initiative has provided proof-of-concept and proof-of-principle for the feasibility of development of a food product in partnership with the food industry. The translation of the diverse research skills and understanding found within a University to the production and successful retail of a food product is one small step towards providing a food environment that is supportive of a healthier diet.

\section{References}

1. Butland, B., Jebb, S., Kopelman, P., et al. Tackling obesities: future choices project report $2^{\text {nd }} E d n$. (2007).

2. Hawkes, C. Food packaging: the medium is the message. (2010) Public Health Nutr 13(2): 297-299.

3. Wang Edward, S.T. The influence of visual packaging design on perceived food product quality, value, and brand preference. (2013) Int J Retail Distrib Manag 41(10): 805-816.

4. Brownell, K.D., Koplan, J.P. Front-of-package nutrition labeling--an abuse of trust by the food industry? The N Engl J Med 364(25): 2373 2375 .

5. Kleef, E.V., Dagevos. H. The growing role of front-of-pack nutrition profile labeling: A consumer perspective on key issues and controversies. (2015) Crit Rev Food Sci 55(3): 291-303.

6. Balasubramanian, S.K., Cole, C. Consumers' search and use of nutrition information: The challenge and promise of the nutrition labeling and education act. (2002) J Marketing 66(3): 112-127. 
7. Mohr, G.S., Lichtenstein, D.R., Janiszewski, C. The effect of marketer-suggested serving size on consumer responses: The unintended consequences of consumer attention to calorie information. (2012) J Marketing 76(1): 59-75.

8. Newman, C.L., Howlett, E., Burton, S. Effects of Objective and Evaluative Front-of-Package Cues on Food Evaluation and Choice: The Moderating Influence of Comparative and Non-Comparative Processing Contexts. (2015) J Consum Res.

9. Dressler, H., Smith, C. Food choice, eating behavior, and food liking differs between lean/normal and overweight/obese, low-income women. (2013) Appetite 65: 145-52.

10. Yan, M.R., Brown, D., Parsons, A., et al. Branding, Ingredients and Nutrition Information: Consumer Liking of a Healthier Snack. (2015) J Food Res 4(5): 64.

11. Australia New Zealand Food Standards Code - Standard 1.2.7 -Nutrition, Health and Related Claims. (2014) Food Standards Australia New Zealand.

12. Martin, K.S., Havens, E., Boyle, K.E., et al. If you stock it, will they buy it? Healthy food availability and customer purchasing behaviour within corner stores in Hartford, CT, USA. (2012) Public Health Nutr 15(10): 1973-1978.
13. Bellisle, F. Meals and snacking, diet quality and energy balance. (2014) Physiol Behav 134: 38-43.

14. Snack Bars in New Zealand. (2015) Euromonitor International.

15. Brown, D. Changing Modal Values through Sustainable Consumption of Food. (2010) AUT University.

16. Doan, A.R., Chambers, E. Predicting success for new flavors with information known pre-launch: A flavored snack food case study. (2012) Food Qual Prefer 25(2): 116-120.

17. Guideline: Sugars intake for adults and children. (2015) World Health Organisation.

18. Bowen, D.J., Barrington, W.E., Beresford, S.A. Identifying the effects of environmental and policy change interventions on healthy eating. (2015) Annu Rev Public Health 36: 289-306.

19. Crofton, E.C., Markey, A., Scannell, A.G. Consumers' expectations and needs towards healthy cereal based snacks: An exploratory study among Irish adults. (2013) Brit Food J 115(8): 1130-1148.

20. McIntyre, C., Baid, A. Indulgent snack experience attributes and healthy choice alternatives. (2009) Brit Food J 111(5): 486-497.

21. vanBuul, V.J., Brouns, F.J. Nutrition and health claims as marketing tools. Crit Rev Food Sci Nutr 55(11): 1552-1560.

22. Buttriss, J.L. Food reformulation: the challenges to the food industry. (2013) Proc Nutr Soc72(1): 61-69. 